\title{
Research Paper \\ Relationship Between Perceived Parenting Styles and Identity Styles With Intensity of Depression in Adolescents
}

\section{Amin Sohrabzadeh Fard', *Banafsheh Gharraee², Ali Asghar Asgharnejad Farid³ ${ }^{3}$ Asma Aghebati}

1. MSc., Department of Clinical Psychology, School of Behavioral Sciences and Mental Health (Tehran Institute of Psychiatry), Iran University of Medical Sciences, Tehran, Iran.

2. PhD of Clinical Psychology, Associate Professor, Department of Clinical Psychology, School of Behavioral Sciences and Mental Health (Tehran Institute of Psychiatry) Iran University of Medical Sciences, Tehran, Iran.

3. PhD of Clinical Psychology, Assistant Professor, Department of Clinical Psychology, School of Behavioral Sciences and Mental Health (Tehran Institute of Psychiatry), Iran University of Medical Sciences, Tehran, Iran.

\begin{tabular}{|c|c|}
\hline $\begin{array}{l}\text { Use vourd device to scan } \\
\text { and read the article online }\end{array}$ & Citation: Sohrabzadeh Fard A. Gharraee B, Asgharneiad Farid AA. Aghebati A. [Relationship Between Perceived Parenting \\
\hline 口f僄回 & $\begin{array}{l}\text { Styles and Identity Styles With Intensity of Depression in Adolescents (Persian)]. Iranian Journal of Psychiatry \& Clinical Psy- } \\
\text { chology. 2017; 22(4):292-299. https://doi.org/10.18869/nirp.ijpcp.22.4.292 }\end{array}$ \\
\hline 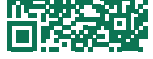 & doi): https://doi.org/10.18869/nirp.ijpcp.22.4.292 \\
\hline
\end{tabular}

Received: 26 Feb. 2016

Accepted: 19 Jun. 2016

Key words:

Parenting, Identity,

Depressive, Ado-

lescent

\section{A B S T R A C T}

Objectives The aim of this study was to examine the relationship between identity styles and dimensions of perceived parenting with the intensity of depression in adolescents.

Methods In a correlation design, 200 (100 girls and 100 boys) students aged 14-18 years were selected from Tehran high schools using cluster sampling. Participants were asked to complete Beck Depression Inventory-2 (BDI-2), Parenting Style Questionnaire (PSQ) and Identity Styles Inventory (ISI) questionnaires. Data were analyzed using Pearson correlation and step-by-step regression (stepwise).

Results Significant relationship was found to exist among the dimensions of perceived parenting and identity style along with depressive intensity. Additionally, predictor variables including identity commitment, dimensions of acceptance, and control parenting with correlation $(r=0.50)$ could predict 0.25 percent of depressive intensity among adolescents $\left(R^{2}=0.52\right)$

Conclusions It seems that identity commitment and parenting style based on parent acceptance among adolescents are two important variables in preventing depressive symptoms. Parenting style and adolescent parent communication are acceptable skills. In addition, the pathology resulting from depression among the adolescents was high, so it is important to address this problem practically.

\section{* Corresponding Author:}

Banafsheh Gharraee, PhD

Address: Department of Clinical Psychology, School of Behavioral Sciences and Mental Health (Tehran Institute of Psychiatry), Iran University of Medical Sciences, Tehran, Iran.

Tel: +98 (21) 66506853

E-mail: gharraee.b@iums.ac.ir 


\title{
ارتباط بين ابعاد فرزنديرورى ادراكشده و سبكهاى هويتى با شدت افسردتى در نوجوانان
}

\author{
امين سهرابزاده فرد'، "بنفشه غرايى"، على اصغر اصغرنزاد فريد"، اسماء عاقبتى"
}

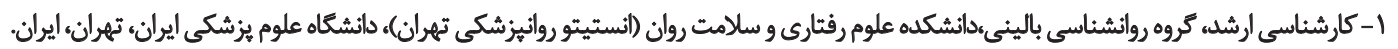

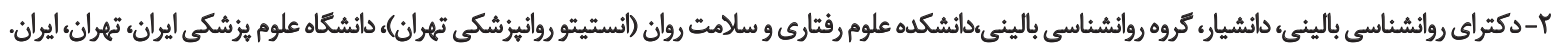

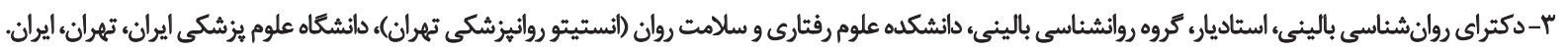

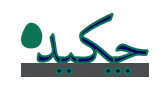

هدف يُروهش حاضر با هدف بررسى رابطه ابعاد فرزئديرورى ادراكشده و سبكهاى هويتى با شدت افسردمى نوجوانان انجام شده است.

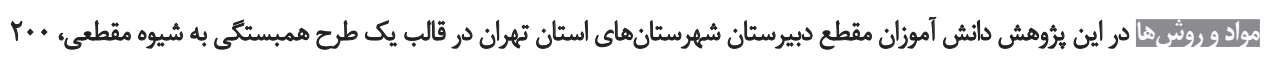

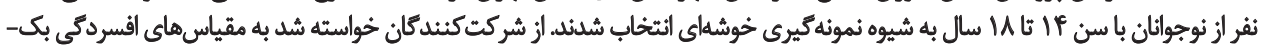

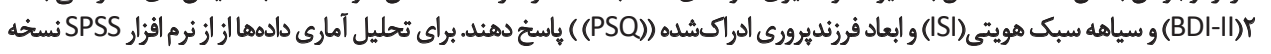

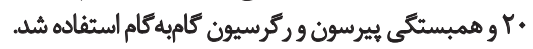

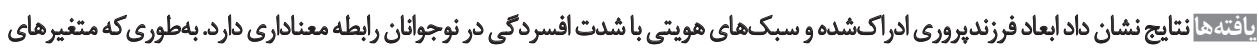

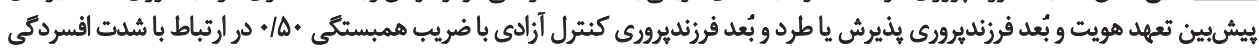

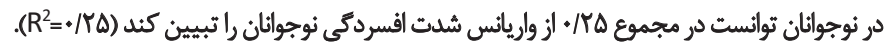

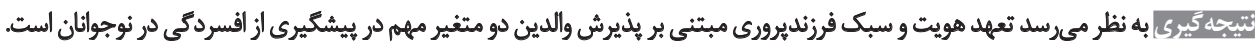

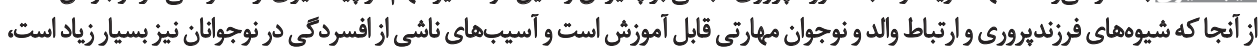
توجه به آموزش اين موضوع در سطح كاربردى اهميت خاصى دارد.
\end{abstract}

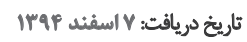

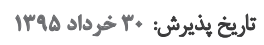

كليدوازهها: - مها:

فرزنديرورى، سبكهاى هويثى، افسردكى، نوجيوانان
والدين و نوجوان افزايش و تعامل كرم بين آنها كاهش مى ميابد.

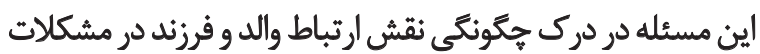

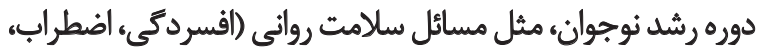

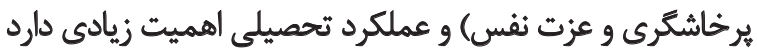

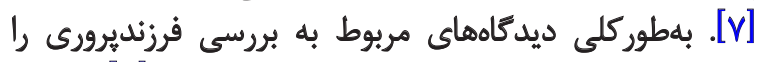

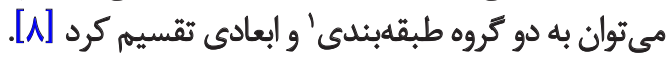
شفرَ (1909) با مطرحكردن ابعاد محبث (طرد و كنترل)

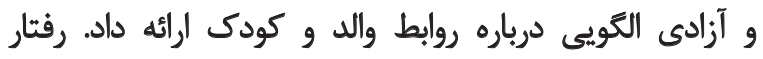

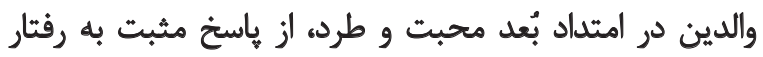

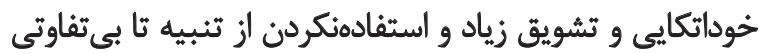

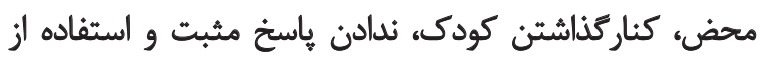
تنبيه متغير است. در امتداد محوركنترل و وآزادى، رفتار ونتيت والدين

1. Typological

2. Shafer
مقدمه نيمى از اختلالات روانى در اواسط نوجوانى آغاز مي شود [1].

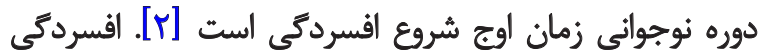

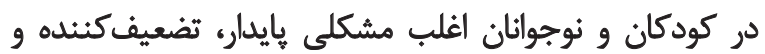

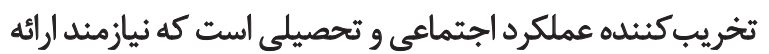

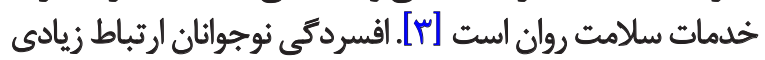

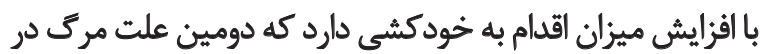

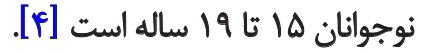

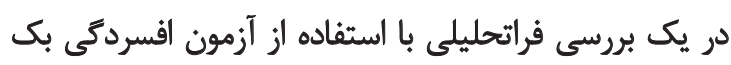

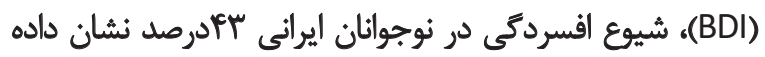

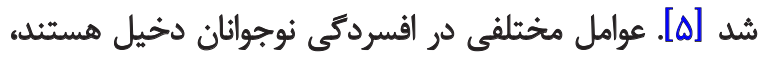

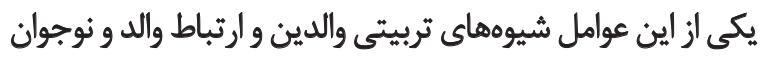

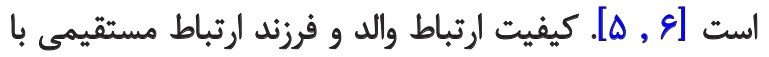

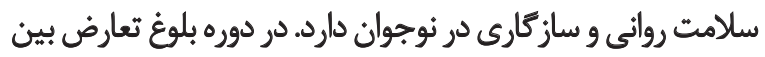




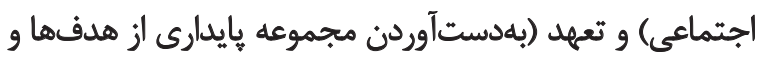

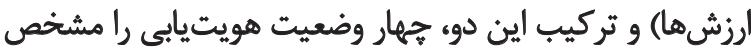

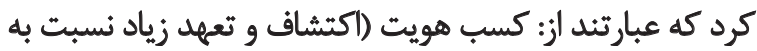

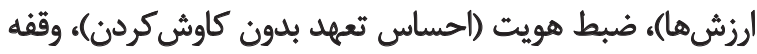

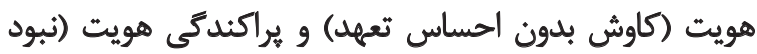

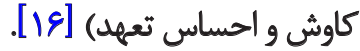

برزونسكى هبر اساس نظريه مارسيا و اريكسون به تدوين

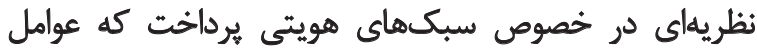

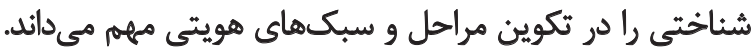

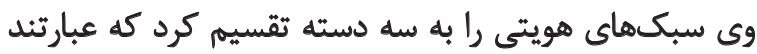

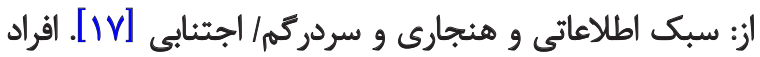

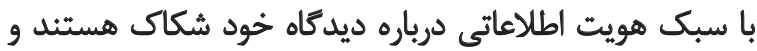

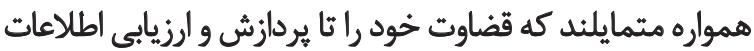

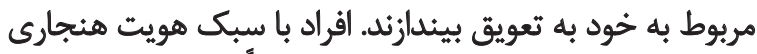

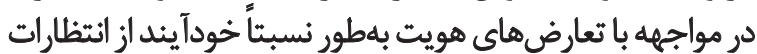

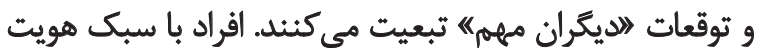

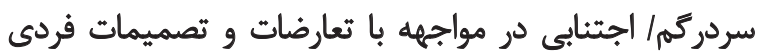

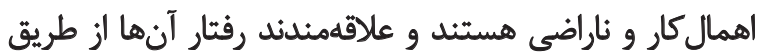

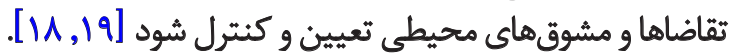

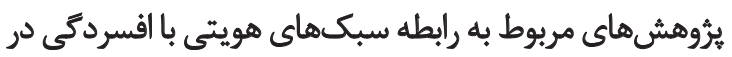

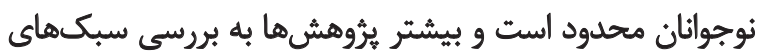

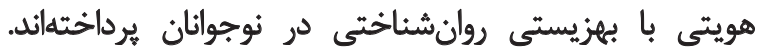

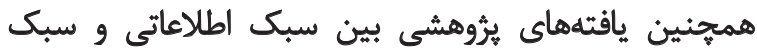

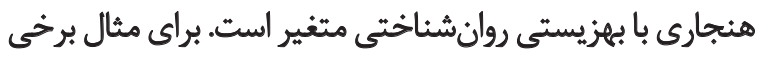

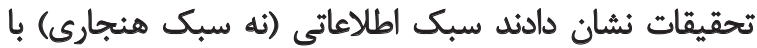

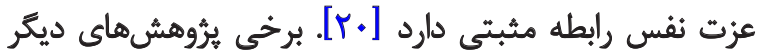

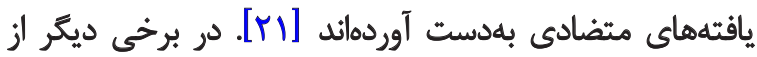

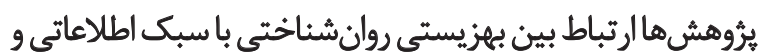

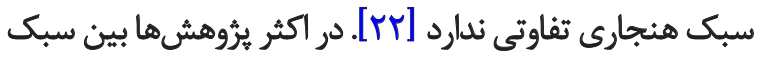

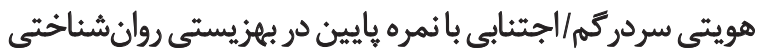

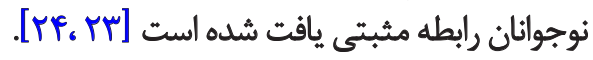

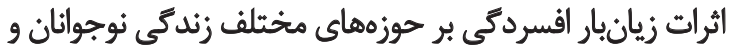

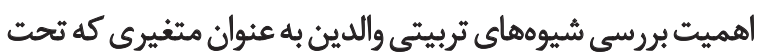

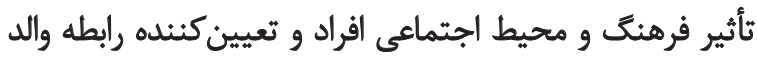

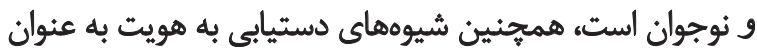

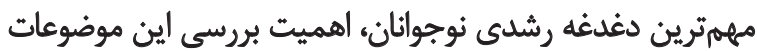

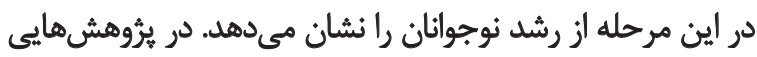

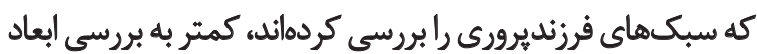
فرزنديرورى برداخته شده است.

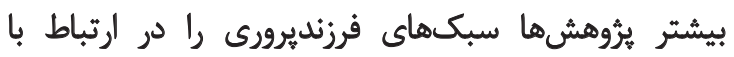

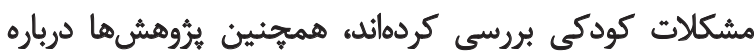

5. Berzonsky

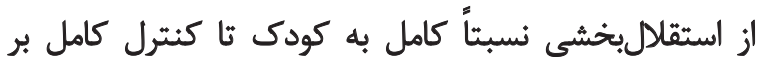

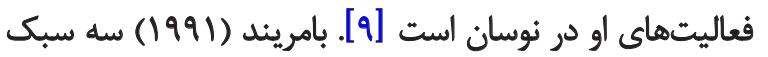

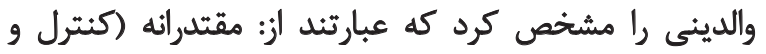

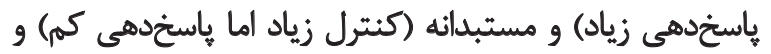
سهل سانكار (كنترل كم اما ياسخدهى زياد) [.1.]. امروزه مدل هاى نظرى بيشتر بر ديدكاه بُعدى تأكيد دارند تأنا

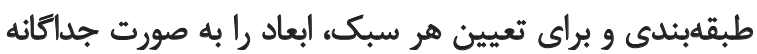

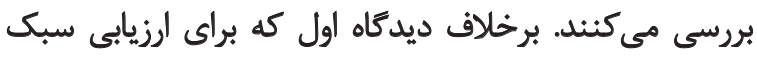

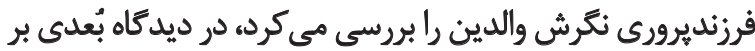

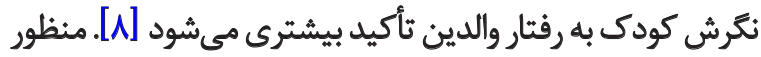

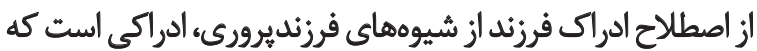

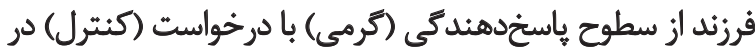

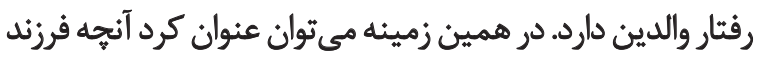

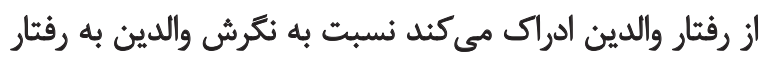
فرزنديرورى خود، اهميت بيشترى دارد [1 1 ، 11]]. در ديدكاه ابعادى مشخص شده است كه رشد مثبت كودكان

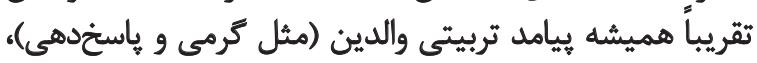

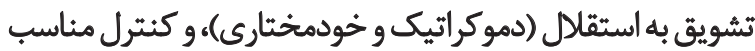

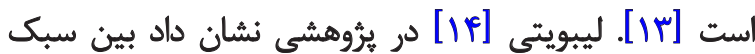

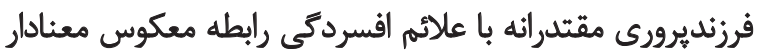

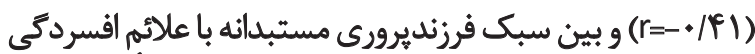

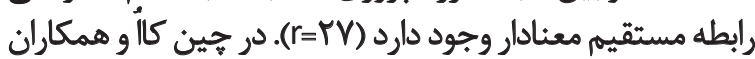

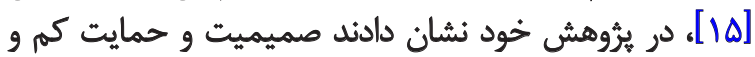

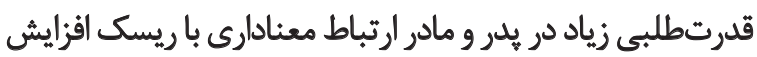

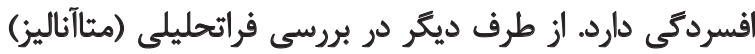

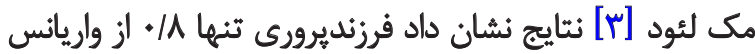

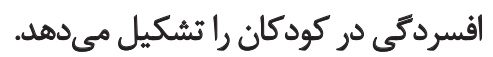

علاوه بر اهميت شيوههاى تربيتى والدين بر سلامت روانى

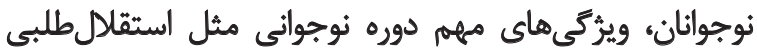

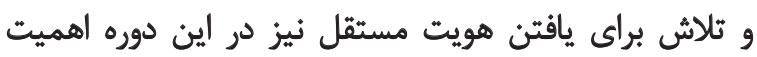

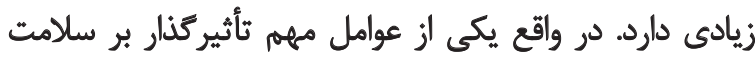

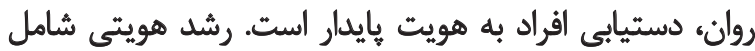

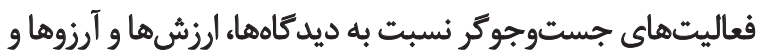
متعهدبودن به آنها در طول زندكي نسي است

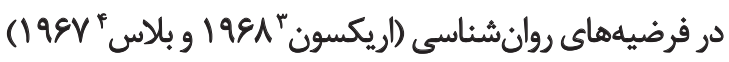

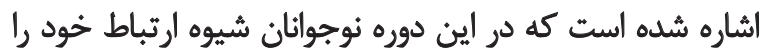

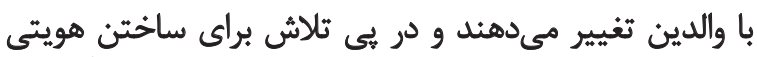

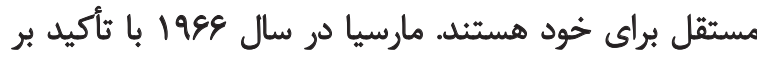

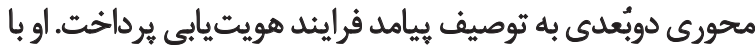
تأكيد بر بُعد اكتشاف (جستوجوى ارزش توحا و باورها و نقشهاي فرائ

\section{Erikson}

4. Blos 


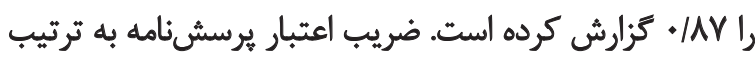

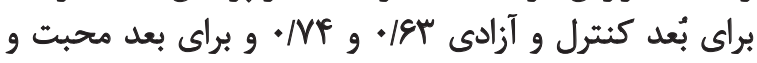

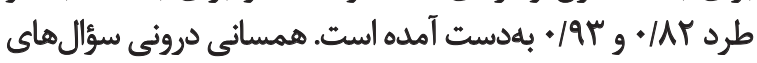

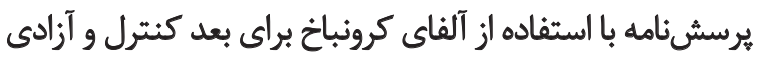

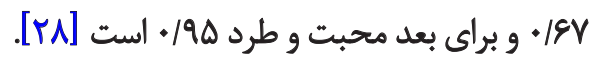

سياهل سبك هويت (ISI)

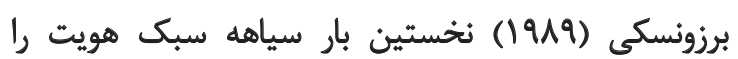

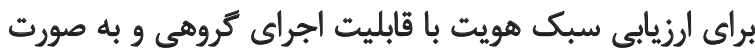

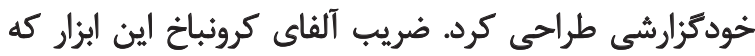

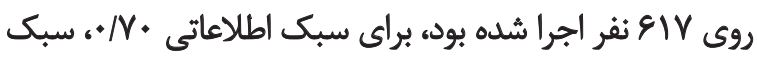

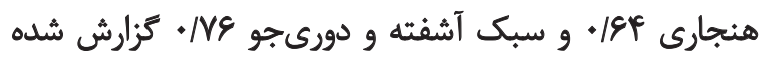

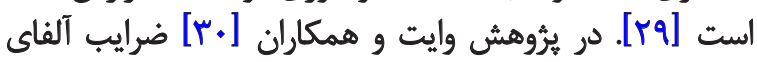

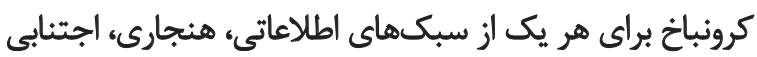

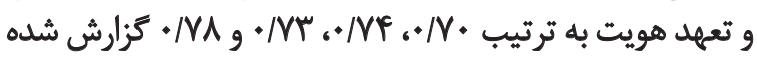

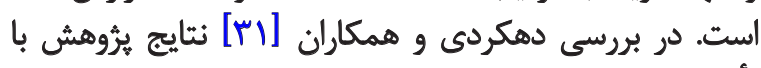

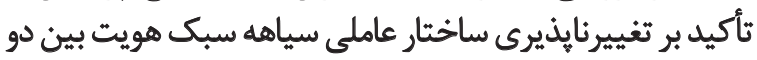

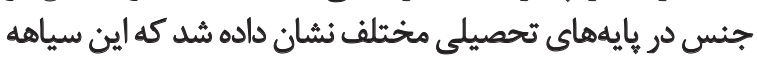

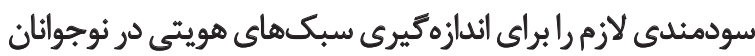

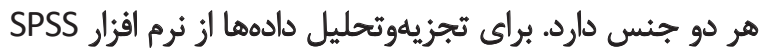

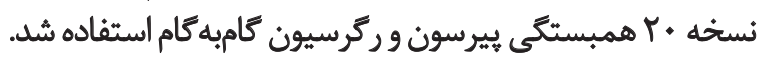

يافتهها

ميانكين كل نمره شدت افسردكى نوجوانان در نمونه

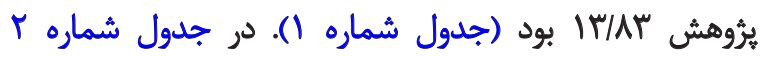

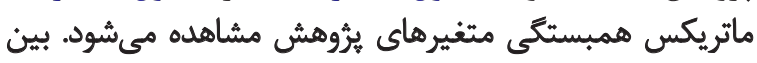

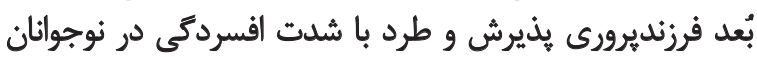

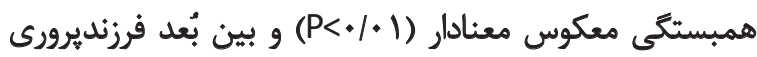

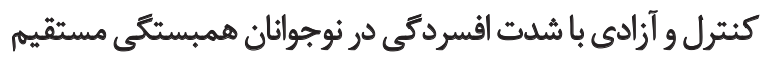

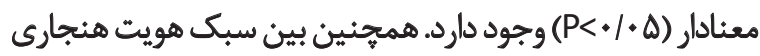

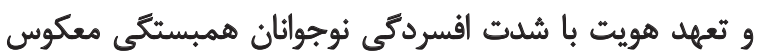

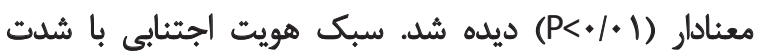

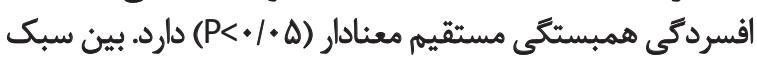
هويت اطلاعاتى با شدت افسردكى رابطه معنادار ديده نشد.

براى بيشبينى شدت افسردكى نوجوانان بر اساس ابعاد

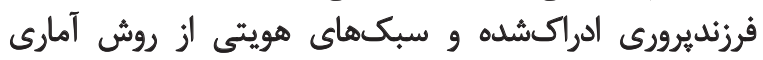

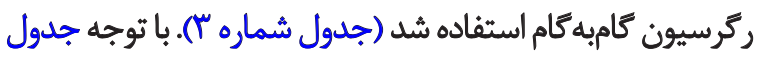

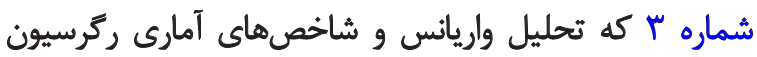

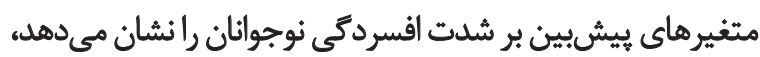

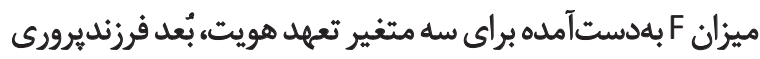
يذيرش و طرد و بُعد فرزنديرورى برى كنترل و آزادى معندادار است.

9. Identity Styles Inventory
ارتباط سبكهاى هويتى با افسردگى نوجوانان بسيار محدود است.

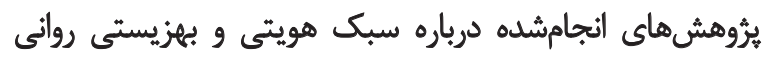

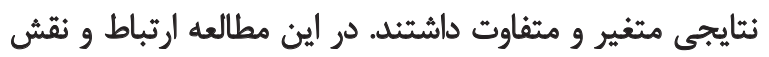

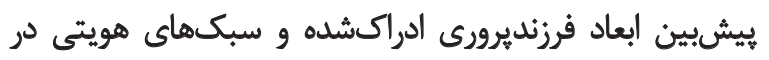
شدت افسردگى نوجوانان بررسى شده است.

ووشي

در ثروهش حاضر از طرح همبستكى به شيوه مقطعى استفاده

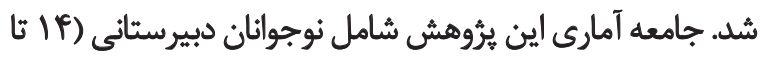

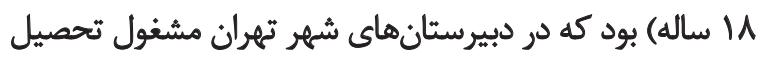

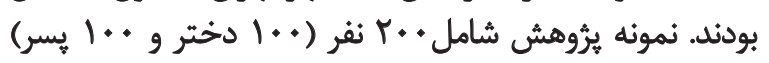

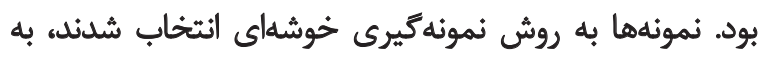

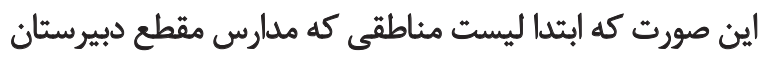

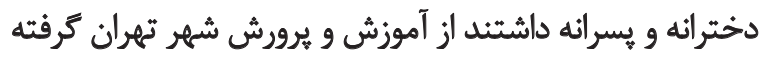

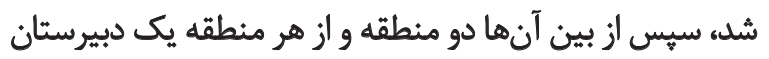

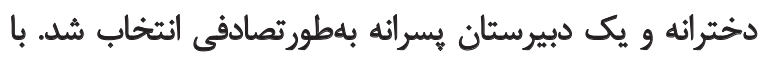

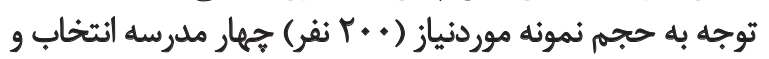

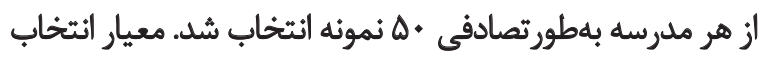

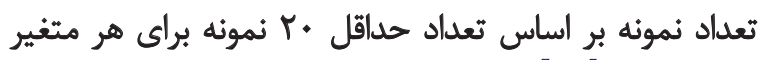

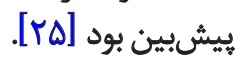

$$
\text { يرسش نامه افسردكى بك - ويرايش دوم ('BDI-II) }
$$

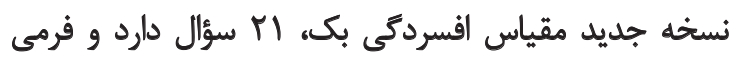

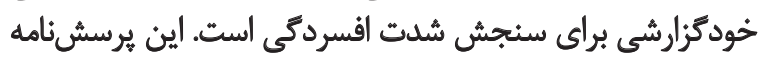

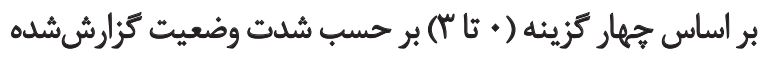

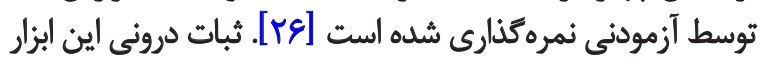

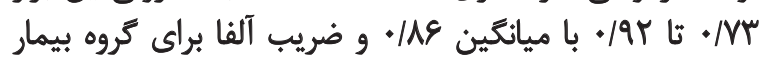

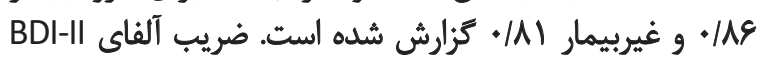

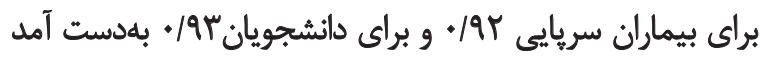

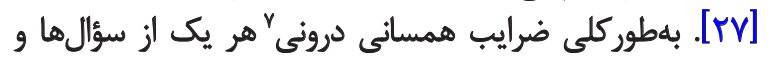

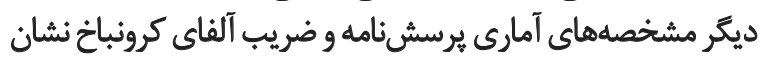

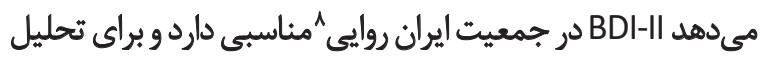

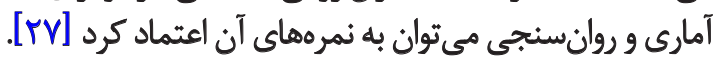

$$
\text { مقياس الدراى از شيوهام فرزئديروىى }
$$

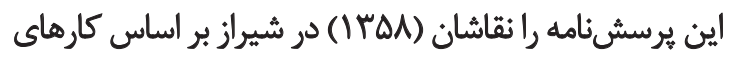

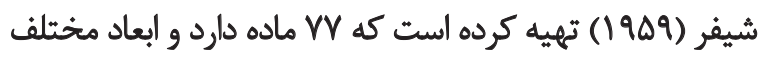

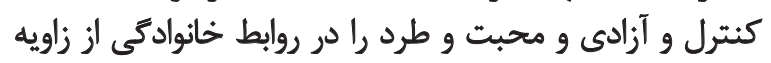

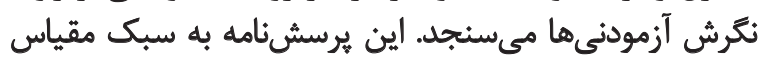

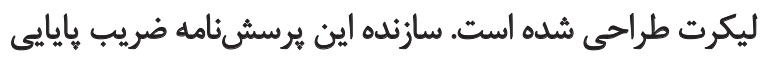

6. Beck Depression Inventory

7. Internal consistency

8. Validity 
جدول ا. ميائتين (و انحراف معيار) هر يك از متغير هاى بثروهش.

\begin{tabular}{|c|c|}
\hline مياتئين (انحراف معيار) & مثغير \\
\hline$($ Nq) Ir/Ar & شلتث افسردكى \\
\hline$(M T / \Delta) I Y N T$ & بعد فرزئد برورى هذيرش -طرد \\
\hline$(I T / q) I M T / m+$ & بعد فرزنديرورى كثترل -Fزادى \\
\hline$(\varepsilon / \Gamma) \pi f / Q$ & سبك هويت اطلاعاتى \\
\hline$(\Delta / \Lambda) T / K$ & سبك هويت هنجارى \\
\hline$(\delta / r) r \Delta / \Delta r$ & سبك هويت اجتنابي \\
\hline$(g / 1) \mathrm{T} / \mathrm{MT}$ & تعهل هويت \\
\hline
\end{tabular}

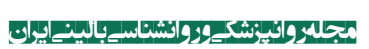

جدول r. ماتريكس همبستكّى متغيرهاى يثروهش.

\begin{tabular}{|c|c|c|c|c|c|c|c|c|}
\hline$v$ & 8 & 0 & $f$ & $r$ & r & 1 & & \\
\hline & & & & & & 1 & 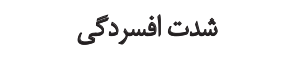 & 1 \\
\hline & & & & & 1 & $-\bullet / \varphi+\cdots$ & بُعد فرزنديرورى يذيرش-طرد & $r$ \\
\hline & & & & 1 & $-\cdot / r$ & .118 & بُعل فرزنديرورى كتترل-آزادى & $r$ \\
\hline & & & 1 &.$/ M r$ &.$/ 19 * *$ & $-* 1+1$ & سبك هويتى اطلاعائي & $p$ \\
\hline & & 1 & $+/ \varphi_{*}^{*}$ & $\cdot / r+*$ & $\cdot / r r^{* *}$ & $-* / M r * *$ & سبك هويتى هنجارى & $\Delta$ \\
\hline & 1 & $-* / 1$ & 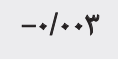 & $\cdot 1 \cdot+r$ & $-+11 \mathrm{~A}^{*}$ &.$/ 10^{\circ}$ & سبك هويتي اجتنايي & $\&$ \\
\hline 1 &.$- / Y q * *$ & $.18 a^{* * *}$ &.$/ \mu_{*}^{* *}$ & $.1 \cdot .+r$ & - prese** & $-\bullet||^{*}$ & تعهل هويت & $v$ \\
\hline
\end{tabular}

افسردكى نوجوانان دارئل با نكاهي به ضرايب بتا مي توان دريافت

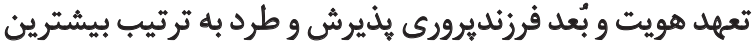

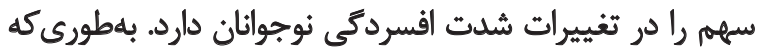

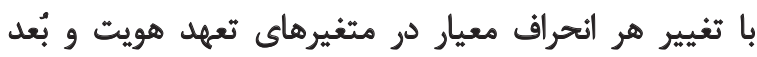

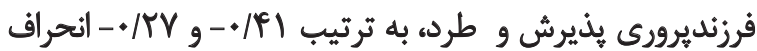

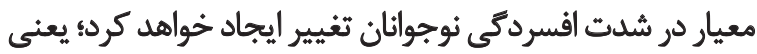
با افزايش ثعهد هويت و سبك فرزنديرورى همراه با هذيرش نواني

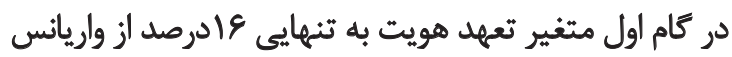

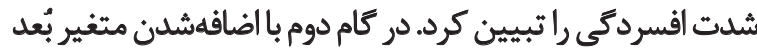

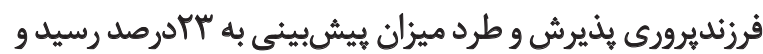

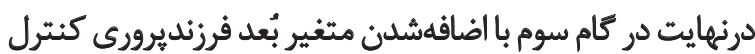

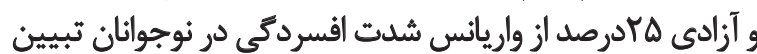

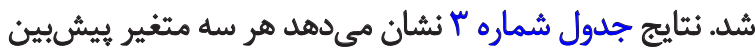

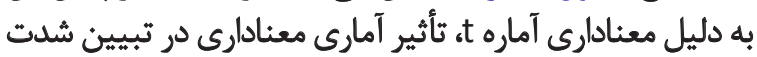

جدول ب. ضرايب ركرسيون و شاخصهاى آمارى ارثباط بين ابعاد فرزئديرورى ادراكشده و سبك هاي هويتى با شدت افسردمي نوجوانان.

\begin{tabular}{|c|c|c|c|c|c|c|c|c|}
\hline $\mathbf{P}$ & $\mathbf{T}$ & $\mathbf{F}$ & Beta & $\mathbf{B}$ & $\mathbf{R}^{2}$ & $\mathbf{R}$ & متغير بيشبين & مدل \\
\hline $.1 .+1$ & $-8 / \mathbb{N}^{2}+\Delta$ & $r+/ N r^{2}$ & $-+/ 41$ &.$- / \Delta 9 \Delta$ &.$/ 18$ & $\cdot \mid+1$ & تعهد هويت & كام اول \\
\hline+1 & $-r / A Y Q$ & raval & $-* / T V$ & $-* M \cdot r$ & ו & - pra & بُعل فرزند يرورى ئذيرش -طرد & كام دوم \\
\hline $.1 . m f$ & $r /|F|$ & $\mathrm{rV} / \mathrm{W}$ & D & $\% N$ &.$/ T \Delta$ & .10 & بُعد فرزند يرورى كنترل-آزادى & 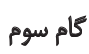 \\
\hline
\end{tabular}


يُروهش در اين موضوع محدود است و بيشتر سبكهاى هويتى برتي در ارتباط با بهزيستى روانشناختى بررسى شده است است با توجه به همبستكى سبك هنجارى با تعهد هويت در اين

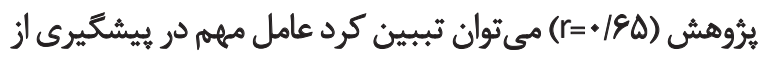

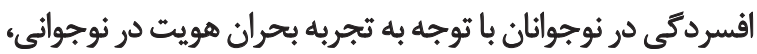

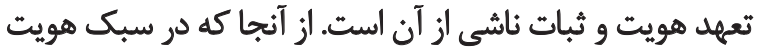

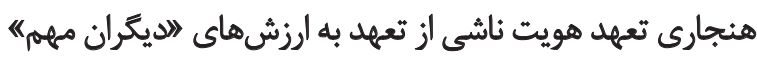

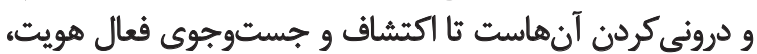

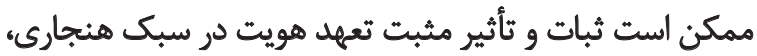

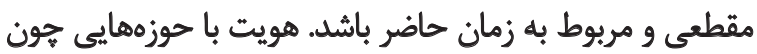

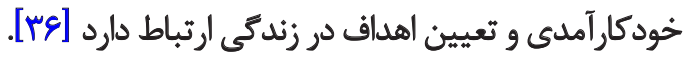

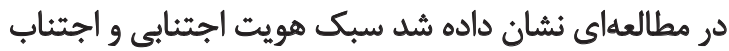

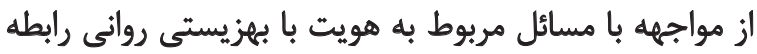

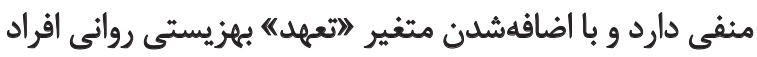

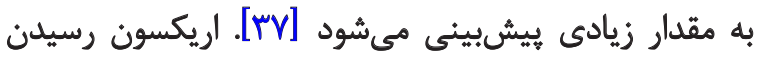

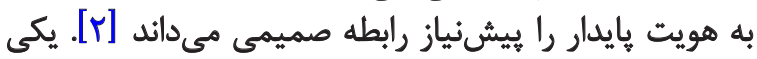

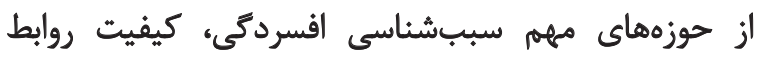

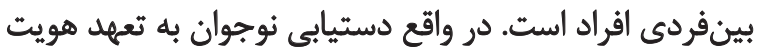

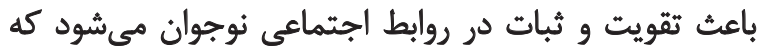

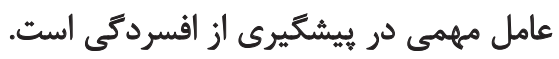

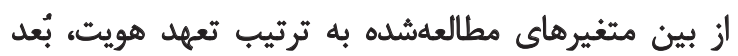

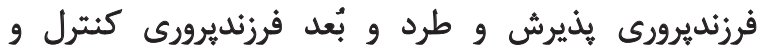

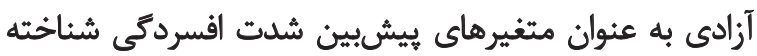

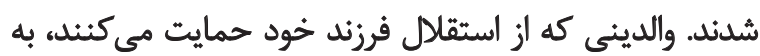

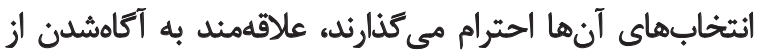

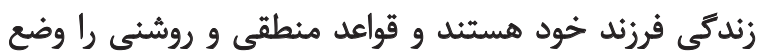

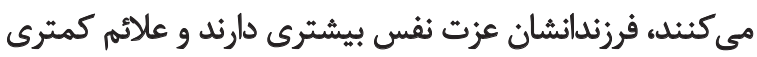

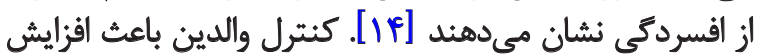

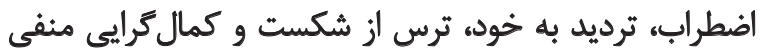

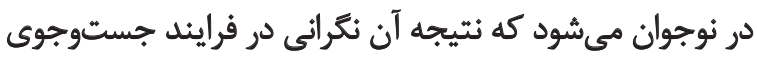

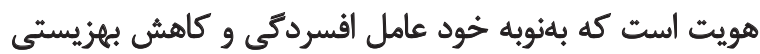

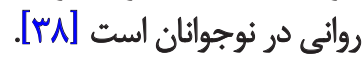

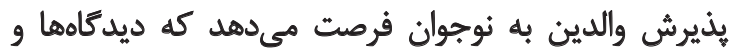

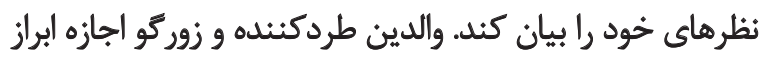

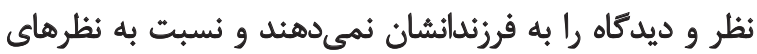

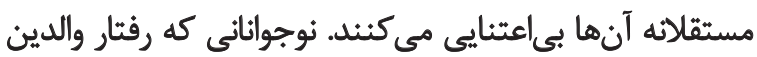

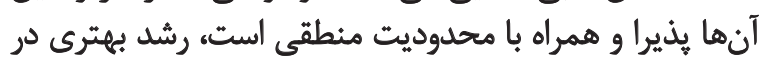

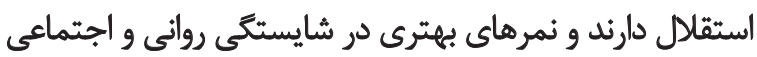

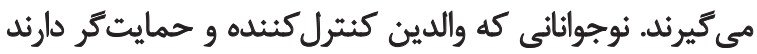

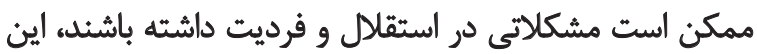

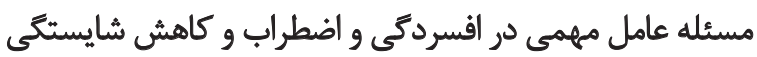
اجتماعي است عامل [ع]
والدين، شدت افسردكى در نوجوانان كاهش مي فيابد. ضريب بئا

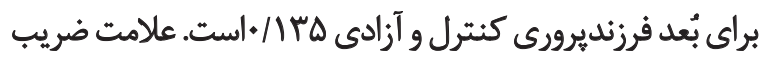

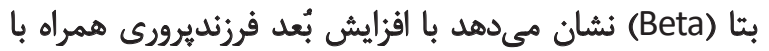
كنترل والدين، شدت افسردخى نوجوانان افزايش مي في فيابد.

بحث

عوامل خانوادكى جزو مهمثرين مولفهاهاى تبيين كنينده منشأ

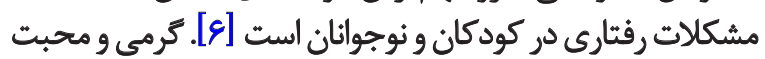

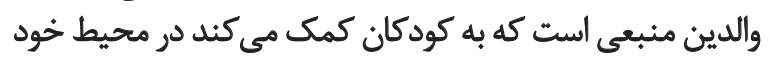

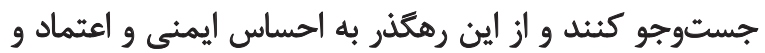

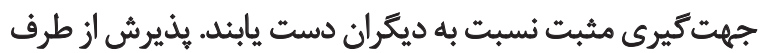

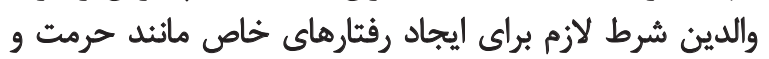

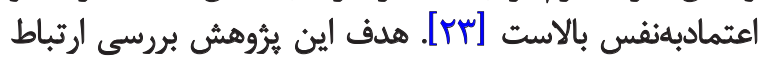

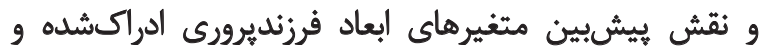

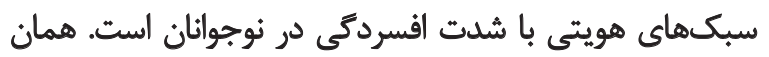

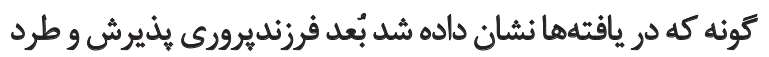

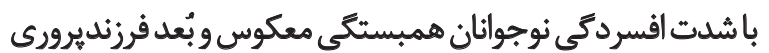

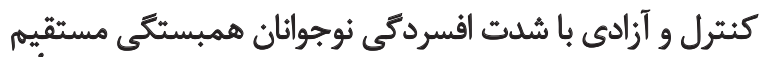

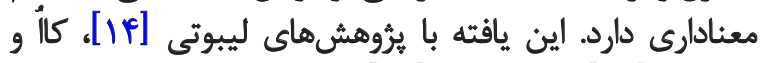

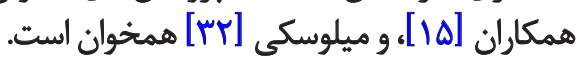

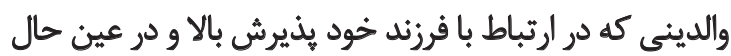

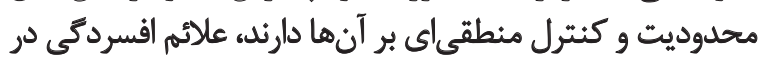

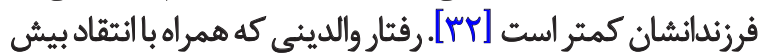

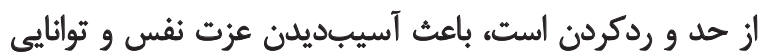

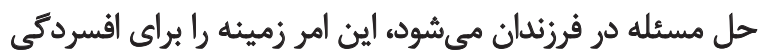

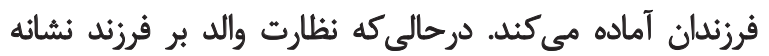

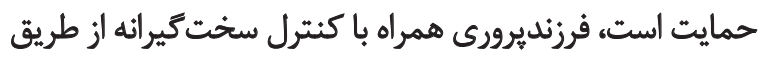

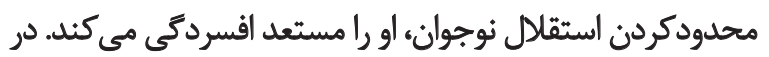

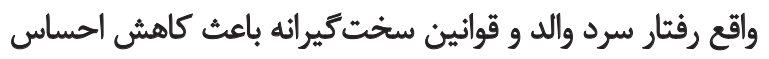

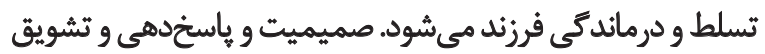

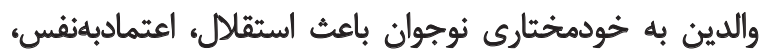

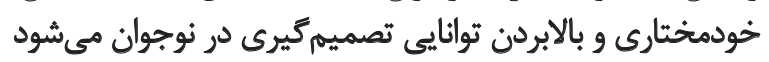

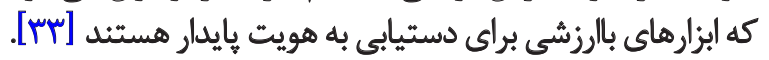

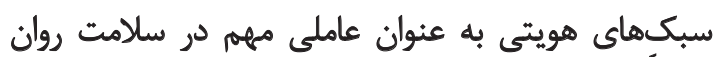

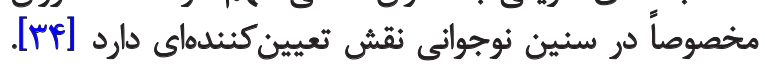

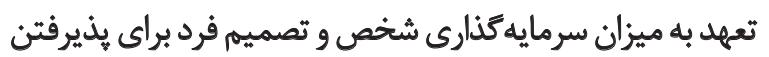

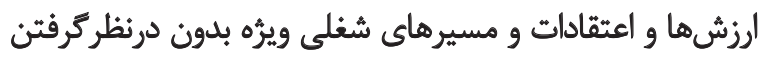

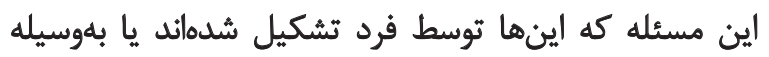

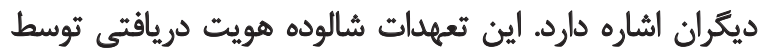

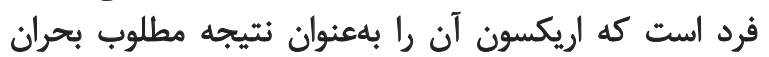

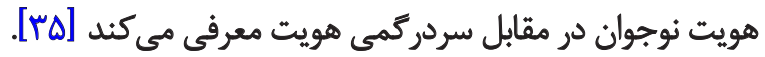

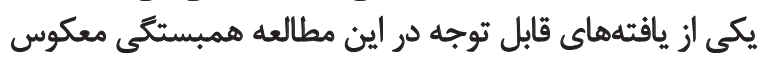
سبك هويت هنجارى با شدت افسردگى نوجوانان است. يُيشينه 


\section{References}

[1] Yap MBH, Pilkington PD, Ryan SM, Jorm AF. Parental factors associated with depression and anxiety in young people: A systematic review and meta-analysis. Journal of Affective Disorders, 2014; 156:8-23. doi: 10.1016/j.jad.2013.11.007

[2] Cairns KE, Yap MBH, Reavley NJ, Jorm AF. Identifying prevention strategies for adolescents to reduce their risk of depression: A Delphi consensus study. Journal of Affective Disorders. 2015; 183:229-38. doi: 10.1016/j.jad.2015.05.019

[3] McLeod BD, Weisz JR, Wood JJ. Examining the association between parenting and childhood depression: A meta-analysis. Clinical Psychology Review. 2007; 27(8):986-1003. doi: 10.1016/j. cpr.2007.03.001

[4] Peden M. World report on child injury prevention. Geneva: World Health Organization; 2008.

[5] Sajjadi H, Mohaqeqi Kamal SH, Rafiey H, Vameghi M, Forouzan AS, Rezaei M. A systematic review of the prevalence and risk factors of depression among Iranian adolescents. Global Journal of Health Science. 2013; 5(3):16-27. doi: 10.5539/gjhs.v5n3p16

[6] Matejevic M, Jovanovic D, Ilic M. Patterns of family functioning and parenting style of adolescents with depressive reactions. Procedia-Social \& Behavioral Sciences. 2015; 185:234-9. doi: 10.1016/j.sbspro.2015.03.460

[7] Smokowski PR, Evans CBR, Cotter KL, Webber KC. Ethnic identity and mental health in American Indian youth: Examining mediation pathways through self-esteem, and future optimism. Journal of Youth \& Adolescence. 2013; 43(3):343-55. doi: 10.1007/ s10964-013-9992-7

[8] Asghari MS, Besharat MA. The relation of perceived parenting with integrative self-knowledge. Procedia-Social \& Behavioral Sciences. 2011; 30:226-30. doi: 10.1016/j.sbspro.2011.10.045

[9] Pellerin LA. Applying Baumrind's parenting typology to high schools: toward a middle-range theory of authoritative socialization. Social Science Research. 2005; 34(2):283-303. doi: 10.1016/j. ssresearch.2004.02.003

[10] Silk JS, Morris AS, Kanaya T, Steinberg L. Psychological control and autonomy granting: Opposite ends of a continuum or distinct constructs? Journal of Research on Adolescence. 2003; 13(1):11328. doi: 10.1111/1532-7795.1301004

[11] Krampen G. Perceived childrearing practices and the development of locus of control in early adolescence. International Journal of Behavioral Development. 1989; 12(2):177-93. doi: $10.1177 / 016502548901200203$

[12] Anlı İ, Karslı TA. Perceived parenting style, depression and anxiety levels in a Turkish late-adolescent population. ProcediaSocial \& Behavioral Sciences. 2010; 2(2):724-7. doi: 10.1016/j.sbspro.2010.03.091

[13] Fan J, Zhang LF. The role of perceived parenting styles in thinking styles. Learning \& Individual Differences. 2014; 32:204-11. doi: 10.1016/j.lindif.2014.03.004

[14] Laboviti B. Perceived parenting styles and their impact on depressive symptoms in adolescent 15-18 years old. Journal of Educational \& Social Research. 2015; 5(1):171-76. doi: 10.5901/ jesr.2015.v5n1p171

\section{تثيجلتيرى}

بيشرفت فناورى و رسائهاي جمعى و وجود دامنه زيادي از

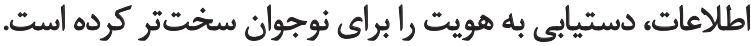

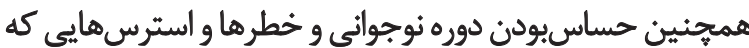

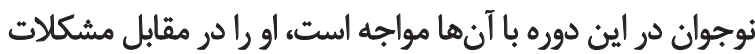

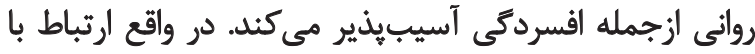

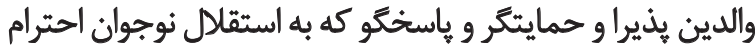

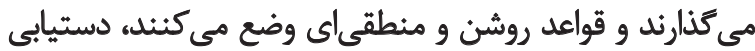

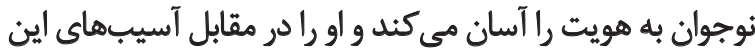

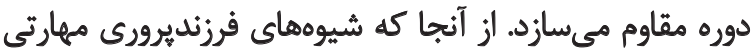

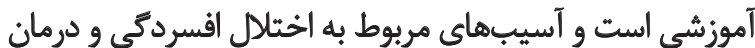

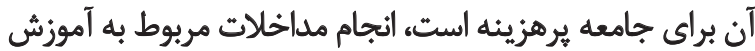

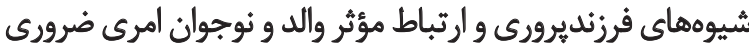

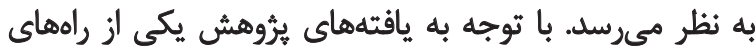

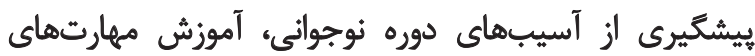

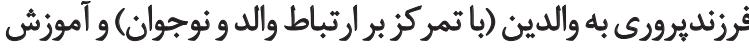

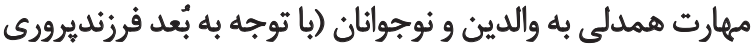

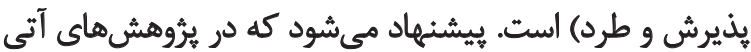

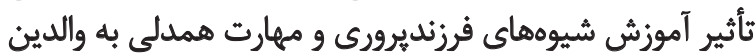
در كاهش افسردگى نوجوانان بررسى شودي

از محدوديتهاى يرُوهش حاضر ميتوان به استفاده از روش

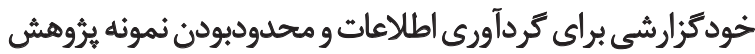

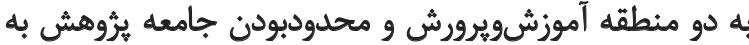

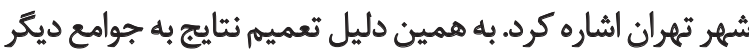

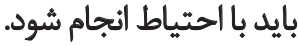

$$
\text { سياستَّزَارى }
$$

از تمام كسانى كه در اين مسير ما را يارى كردند، مخصوصاً

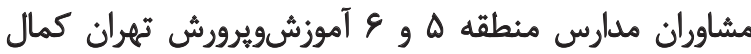

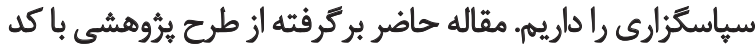

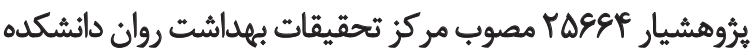
علوم رفتارى و سلامت روان ( انستيتو روانيزشكى تهران بران) دانشعًاه علوم يزشكى ايران است. إين مقاله بركرفتها إيايان نامه مقطع كارشناسى ارشد نويسنده

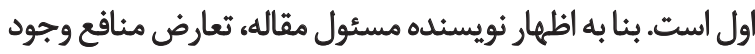

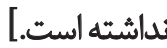


[15] Gao J, Li Y, Cai Y, Chen J, Shen Y, Ni S, et al. Perceived parenting and risk for major depression in Chinese women. Psychological Medicine. 2012; 42(5):921-30. doi: 10.1017/s0033291711001942

[16] Kroger J, Martinussen M, Marcia JE. Identity status change during adolescence and young adulthood: A meta-analysis. Journal of Adolescence. 2010; 33(5):683-98. doi: 10.1016/j.adolescence.2009.11.002

[17] Berzonsky MD, Cieciuch J, Duriez B, Soenens B. The how and what of identity formation: Associations between identity styles and value orientations. Personality \& Individual Differences. 2011; 50(2):295-9. doi: 10.1016/j.paid.2010.10.007

[18] Berzonsky MD. Identity formation: The role of identity processing style and cognitive processes. Personality \& Individual Differences. 2008; 44(3):645-55. doi: 10.1016/j.paid.2007.09.024

[19] Berzonsky MD, Kuk LS. Identity style, psychosocial maturity, and academic performance. Personality \& Individual Differences. 2005; 39(1):235-47. doi: 10.1016/j.paid.2005.01.010

[20] Crocetti E, Rubini M, Meeus W. Capturing the dynamics of identity formation in various ethnic groups: Development and validation of a three-dimensional model. Journal of Adolescence. 2008; 31(2):207-22. doi: 10.1016/j.adolescence.2007.09.002

[21] Johnson EA, Nozick KJ. Personality, adjustment, and identity style influences on stability in identity and self-concept during the transition to university. Identity: An International Journal of Theory \& Research. 2011; 11(1):25-46. doi: 10.1080/15283488.2011.540737

[22] Beaumont SL, Zukanovic R. Identity development in men and its relation to psychosocial distress and self-worth. Canadian Journal of Behavioural Science. 2005; 37(1):70-81. doi: 10.1037/ h0087246

[23] Pellerone M, Spinelloa C, Sidoti A, Micciche S. Identity, perception of parent-adolescent relation and adjustment in a group of university students. Procedia-Social \& Behavioral Sciences. 2015;190:459-64. doio: 10.1016/j.sbspro.2015.05.026

[24] Phillips TM, Pittman JF. Adolescent psychological well-being by identity style. Journal of Adolescence. 2007; 30(6):1021-34. doi: 10.1016/j.adolescence.2007.03.002

[25] Jewell NP. Statistics for epidemiology. Philadelphia: CRC Press, Taylor \& Francis Group; 2003.

[26] Beck AT, Guth D, Steer RA, Ball R. Screening for major depression disorders in medical inpatients with the Beck Depression Inventory for Primary Care. Behaviour Research \& Therapy. 1997; 35(8):785-91. doi: 10.1016/s0005-7967(97)00025-9

[27] Khoshnam S, Borjali A, Karegari Padar L, Amiri H. [Effectiveness of emotion-focused therapy on patients with major depression disorder (Persian)]. Journal of Contemporary Psychology. 2014; 9(1):95-106.

[28] Pakdaman S, Khanjani M. [The role of perceived parenting in the relationship between attachment and collectivism styles among university students (Persian)]. Journal of Social Psychology Research. 2012; 1(4):81-102.

[29] Berzonsky MD, Kuk LS. Identity style, psychological Maturity, and academic performance. Personality \& Individual Differences. 2005; 39(1):235-247. doi: 10.1016/j.paid.2005.01.010

[30] White JM, Wampler RS, Winn KI. The identity style inventory: A revision with a sixth-grade reading level (ISI-
6G). Journal of Adolescent Research. 1998; 13(2):223-45. doi: $10.1177 / 0743554898132007$

[31] Aliakbari Dehkordi M, Khodaei A, Shokri O, Daneshvarpoor Z. [Cross-group invariance of factorial structure of the identity style inventory among Iranian adolescents (Persian)]. Journal of Developmental Psychology: Iranian Psychologist. 2013; 9(35):237-248.

[32] Milevsky A, Schlechter M, Netter S. Maternal and paternal parenting styles in adolescents: associations with self-esteem, depression and life-satisfaction. Journal of Child \& Family Studies. 2007, 16(1):39-47. doi: 10.1007/s10826-006-9066-5

[33] Smits I, Soenens B, Luyckx K, Duriez B, Berzonsky M, Goossens L. Perceived parenting dimensions and identity styles: Exploring the socialization of adolescents' processing of identity-relevant information. Journal of Adolescence. 2008; 31(2):151-64.doi: 10.1016/j.adolescence.2007.08.007

[34] Phillips TM, Pittman JF. Adolescent psychological well-being by identity style. Journal of Adolescence. 2007; 30(6):1021-34. doi: 10.1016/j.adolescence.2007.03.002

[35] White RJ. The role of parenting style, ethnicity, and identity style on identity commitment and career decision self-efficacy [PhD thesis]. California: University of Southern California; 2009.

[36] Ryan RM, Deci EL. On assimilating identities to the self: A selfdetermination theory perspective on internalization and integrity within cultures. New York: Guilford Press; 2003.

[37] Shokri O, Tajik Esmaeili, Daneshvarpoor Z, Ghanaei Z, Dastjerdi R. [Individual differences in identity styles and psychological rehabilitation: the role of identity commitment (Persian)]. Advances in Cognitive Science. 2007; 9(2):33-46

[38] Pesigan IJA, Luyckx K, Alampay LP. Brief report: Identity processes in Filipino late adolescents and young adults: Parental influences and mental health outcomes. Journal of Adolescence. 2014; 37(5):599-604. doi: 10.1016/j.adolescence.2014.04.012 